\title{
One loop renormalization of the Littlest Higgs model
}

\author{
Benjamín Grinstein, ${ }^{a, b}$ Randall Kelley ${ }^{c}$ and Patipan Uttayarat ${ }^{a}$ \\ ${ }^{a}$ Department of Physics, University of California at San Diego, \\ La Jolla, CA 92093, U.S.A. \\ ${ }^{b}$ Theory Division, CERN, CH-1211 Geneva 23, Switzerland \\ ${ }^{c}$ Center for the Fundamental Laws of Nature, Harvard University, \\ Cambridge, MA 02138, U.S.A. \\ E-mail: bgrinstein@ucsd.edu, randallkelley@physics.harvard.edu, \\ puttayarat@physics.ucsd.edu
}

ABSTRACT: In Little Higgs models a collective symmetry prevents the Higgs from acquiring a quadratically divergent mass at one loop. This collective symmetry is broken by weakly gauged interactions. Terms, like Yukawa couplings, that display collective symmetry in the bare Lagrangian are generically renormalized into a sum of terms that do not respect the collective symmetry except possibly at one renormalization point where the couplings are related so that the symmetry is restored. We study here the one loop renormalization of a prototypical example, the Littlest Higgs Model. Some features of the renormalization of this model are novel, unfamiliar form similar chiral Lagrangian studies.

Keywords: Beyond Standard Model, Higgs Physics, Renormalization Group

ARXIV EPRINT: 1011.0682 


\section{Contents}

1 Introduction 1

2 The model 2

3 General structure of counterterms $\quad 5$

3.1 Scalar kinetic energy counterterms 5

3.1.1 Scalar kinetic counterterms from gauge interaction 6

$\begin{array}{lll}\text { 3.1.2 Scalar kinetic counterterms from Yukawa interaction } & 7\end{array}$

$\begin{array}{lll}3.2 & \text { Fermion kinetic energy counterterms } & 8\end{array}$

$\begin{array}{lll}3.3 & \text { Yukawa vertex counterterms } & 9\end{array}$

3.4 Counterterms to counterterms: the general case 9

4 Renormalization $\quad 10$

$\begin{array}{llr}4.1 & \text { Generalities } & 10\end{array}$

4.2 Matching counterterms $\quad 11$

4.2.1 Scalar 2-Point functions with arbitrary scalar background $\quad 11$

$\begin{array}{lll}4.2 .2 & \text { Fermion 2-point functions } & 14\end{array}$

$\begin{array}{lll}4.2 .3 & \text { Yukawa vertex counterterms } & 14\end{array}$

$\begin{array}{lll}4.3 & \beta \text { functions } & 15\end{array}$

5 Conclusions $\quad 16$

\section{Introduction}

The Littlest Higgs $\left(\mathrm{L}^{2} \mathrm{H}\right)$ model [1] is a realization of the idea that the Higgs field, responsible for electroweak symmetry breaking, is a pseudo-Goldstone boson, and as such its mass is automatically small (for some reviews see ref. [2-4]). What is meant by "small" is that the Higgs mass can be made arbitrarily small compared to the scale of breaking of the symmetry that gives rise to this Goldstone boson. Earlier realizations of this idea faced difficulties, required additional fine tuning $[5,6]$. In the $\mathrm{L}^{2} \mathrm{H}$ model, as well as its many extensions, the absence of quadratically divergent radiative corrections to the Higgs mass is guaranteed, at one loop order, by the collective symmetry argument. The argument fails beyond one loop order, so the Higgs can be made naturally light only if its mass is no smaller than of the order of a two loop radiative correction with a cut-off at the scale of the new physics.

While there is a vast literature exploring the phenomenological effects of $\mathrm{L}^{2} \mathrm{H}$-type models, the renormalization structure of the model has been little explored. Computations have been presented that check that the collective symmetry argument does work; however, the structure of counterterms needed to subtract the divergences that do occur has not been studied. Furthermore, the renormalization group equations have not been determined. 
Phenomenologically the $\mathrm{L}^{2} \mathrm{H}$ model has fallen somewhat out of favor because of its difficulties simultaneously accommodating the electroweak precision constraints and in solving the little hierarchy problem [7-11]. However, its structure is prototypical of many models, like Littlest Higgs models with reduced gauge symmetry [20], or with custodial [12, 13] or T-parity [14-16] symmetries. Therefore, the methods we will introduce here should be directly applicable to the one loop renormalization of any of the models in this class.

It was noted in ref. [17] that renormalization group running of the top Yukawa coupling in $\mathrm{L}^{2} \mathrm{H}$-models disrupts the collective symmetry. That is, in order for the collective symmetry argument to operate in the top-quark Yukawa sector, the coupling is built to satisfy an $\mathrm{SU}(3)$ symmetry. However, this symmetry is broken by weak gauge interactions. The would be $\mathrm{SU}(3)$ symmetric top-Yukawa coupling actually splits into two $\mathrm{SU}(2) \times \mathrm{U}(1)$ symmetric terms with coupling constants that run away from each other as they evolve under the renormalization group. This begs the question, what is the full renormalization group structure of the model? It is the purpose of this paper to address this question, at one loop order.

There are several energy scales associated with this model. In addition to the cutoff, $\Lambda$, there is the scale of masses of heavy vector bosons, $g f$ where $f \sim \Lambda / 4 \pi$ is a Goldstone boson decay constant and $g$ some gauge coupling, and the electroweak breaking scale $v$. We are largely interested in the cut-off dependence, so for our computations we will focus on the largest energies, above $g f$. Therefore to determine the ultraviolet behavior we retain the massive gauge vector bosons in our calculations and neglect their masses. On the other hand, the renormalization structure below the scale of these masses, $g f$, is well understood. The model reduces there to the standard electroweak model with one Higgs doublet supplemented by irrelevant operators.

The main result of this paper, the splitting of the Yukawa couplings responsible for the top quark mass, was already noted in ref. [17]. There, a no-go theorem for the collective symmetry mechanism for Yukawa terms was proved. However, the details of the calculation of the running of Yukawa couplings were not given there since, as can be seen from this work, this merits a lengthy discussion that would have detracted from its main point. In fact, we have encountered several stumbling blocks, and corresponding solutions, along the way. Readers interested in questions of principle or practice, or both, in $\mathrm{L}^{2} \mathrm{H}$-type models, will hopefully find this work useful.

The paper is organized as follows. We first review the $\mathrm{L}^{2} \mathrm{H}$ Model in section 2. The 1loop non-derivative counterterms formed only of scalar fields has been extensively studied in the context of determining the effective potential. We classify the remaining counterterms needed to renormalize the model at one loop in section 3 and proceed to compute the renormalization constants and corresponding beta functions in section 4 . We offer some brief concluding remarks in section 5 .

\section{The model}

The $\mathrm{L}^{2} \mathrm{H}$ model is an effective low energy description of some incompletely specified shorter distance dynamics. The short distance dynamics has a global "flavor" symmetry $G_{f}=$ $\mathrm{SU}(5)$, of which a subgroup $G_{w}=\mathrm{SU}(2) \times \mathrm{SU}(2) \times \mathrm{U}(1) \times \mathrm{U}(1)$ is weakly gauged. In the absence of this weak gauge force, the flavor symmetry is broken spontaneously to a 
subgroup $H=\mathrm{SO}(5)$ due to hyper-strong interactions at a scale $\Lambda$. As a result, there are massless Goldstone bosons that are coordinates on the $G_{f} / H$ coset space. Since the weakly gauged $G_{w}$ force breaks the flavor symmetry explicitly, including its effects leads to some of the Goldstone bosons (the would-be Goldstone bosons) being eaten by the Higgs mechanism and the rest becoming pseudo-Goldstone bosons (PGBs) acquiring small masses of order $\Lambda$ times a small symmetry breaking parameter, a gauge coupling constant of the weakly gauged $G_{w}$. The Higgs is the lightest PGB in Little Higgs models, and its mass is naturally much less than $\Lambda$ (and the other PGBs): due to the collective symmetry breaking mechanism a contribution of order $\Lambda^{2}$ to its mass arises only at two loops.

To establish notation we briefly review elements of the $\mathrm{L}^{2} \mathrm{H}$. Symmetry breaking $\mathrm{SU}(5) \rightarrow \mathrm{SO}(5)$ is characterized by the Goldstone boson decay constant $f$. The embedding of $G_{w}$ in $G_{f}$ is fixed by taking the gauge generators

$$
\begin{aligned}
& Q_{1}^{a}=\left(\begin{array}{cccc}
\tau^{a} / 2 & 0 & 0 \\
0 & 0 & 0 \\
0 & 0 & 0
\end{array}\right), \quad Y_{1}=\operatorname{diag}(3,3,-2,-2,-2) / 10 \\
& Q_{2}^{a}=\left(\begin{array}{ccc}
0 & 0 & 0 \\
0 & 0 & 0 \\
0 & 0 & -\tau^{a *} / 2
\end{array}\right), \quad Y_{2}=\operatorname{diag}(2,2,2,-3,-3) / 10
\end{aligned}
$$

The vacuum manifold is characterized by a unitary, symmetric $5 \times 5$ matrix $\Sigma$, transforming as $\Sigma \rightarrow U \Sigma U^{T}$ under $U \in \mathrm{SU}(5)$. A convenient parametrization of $\Sigma$ in terms of the hermitian matrix of Goldstone bosons $\Pi$ is

$$
\Sigma=e^{2 i \Pi / f} \Sigma_{0}, \quad \Sigma_{0}=\left(\begin{array}{ccc}
0 & 0 & \mathbb{1}_{2 \times 2} \\
0 & 1 & 0 \\
\mathbb{1}_{2 \times 2} & 0 & 0
\end{array}\right)
$$

where

$$
\Pi=\left(\begin{array}{ccc}
\omega+\eta \mathbb{1} / \sqrt{20} & h / \sqrt{2} & \phi \\
h^{\dagger} / \sqrt{2} & -2 \eta / \sqrt{5} & h^{T} / \sqrt{2} \\
\phi^{*} & h^{*} / \sqrt{2} & \omega^{T}+\eta \mathbb{1} / \sqrt{20}
\end{array}\right)
$$

Here $\Sigma_{0}$ gives the dynamically determined direction in which the vacuum aligns ${ }^{1}[18,19]$ relative to the embedding of $G_{w}$ in $G_{f}$ given in eq. (2.1). Fluctuations along broken symmetry directions are parametrized by fourteen fields in $\Pi$ : $\omega$ and $\phi$ are $2 \times 2$ matrices satisfying $\omega^{\dagger}=\omega$ and $\phi^{T}=\phi, h$ is an unrestricted $2 \times 1$ matrix and $\eta$ is $1 \times 1$ and real. The vacuum spontaneously breaks $G_{w} \rightarrow \mathrm{SU}(2) \times \mathrm{U}(1)$, and the four fields in $\omega$ and $\eta$ are eaten by the broken generators of gauge symmetries.

The covariant derivative is

$$
D_{\mu} \Sigma=\partial_{\mu} \Sigma-i \sum_{j=1}^{2}\left[g_{j} W_{j \mu}^{a}\left(Q_{j}^{a} \Sigma+\Sigma Q_{j}^{a T}\right)+g_{j}^{\prime} B_{j \mu}\left(Y_{j} \Sigma+\Sigma Y_{j}\right)\right],
$$

\footnotetext{
${ }^{1}$ To ensure this alignment the weakly gauge coupling constant have to be strong enough; see ref. [21].
} 
where $B_{j}$ and $W_{j}^{a}$ are the $\mathrm{U}(1)_{j}$ and $\mathrm{SU}(2)_{j}$ gauge fields respectively. The $\mathrm{U}(1)_{j}$ coupling constant is taken to be $g_{j}^{\prime}$ while the $\mathrm{SU}(2)_{j}$ coupling constant is $g_{j}$.

The effective low energy theory has kinetic term

$$
\mathcal{L}_{\text {kin }}=\frac{f^{2}}{8} \operatorname{Tr}\left(D_{\mu} \Sigma\right)\left(D^{\mu} \Sigma\right)^{\dagger} .
$$

If one sets $g_{1}=g_{1}^{\prime}=0$ the model has an exact global SU(3) symmetry (acting on upper $3 \times 3$ block of $\Sigma$ ), while for $g_{2}=g_{2}^{\prime}=0$ it has a different exact global SU(3) symmetry (acting on the lower $3 \times 3$ block). Either of these exact global SU(3) would-be symmetries guarantee the Higgs remains exactly massless. Hence, the Higgs mass should vanish for either $g_{1}=g_{1}^{\prime}=0$ or $g_{2}=g_{2}^{\prime}=0$. The perturbative quadratically divergent correction to the Higgs mass must be polynomial in the couplings and can involve only one of the couplings at a time at one loop order. Hence it must vanish at one loop. This is the collective symmetry mechanism that ensures the absence of 1-loop quadratic divergences in the Higgs mass.

For a top-quark sector introduce a pair of singlet Weyl fermions $u_{L}$ and $u_{R}$ with hypercharge 2/3. $u_{L}$ is combined with the $3^{\text {rd }}$ generation doublet $q_{L}=\left(t_{L}, b_{L}\right)^{T}$ to form a "royal" triplet

$$
\chi_{L}=\left(\begin{array}{c}
i \tau^{2} q_{L} \\
u_{L}
\end{array}\right) .
$$

The top Yukawa interaction is obtained from coupling the fermions to the upper right $2 \times 3$ block of the $\Sigma$ field,

$$
\mathcal{L}_{\text {top }}=-\frac{1}{2} \lambda_{1} f \bar{\chi}_{L I} \epsilon^{I J K} \epsilon^{x y} \Sigma_{J x} \Sigma_{K y} q_{R}-\lambda_{2} f \bar{u}_{L} u_{R}+\text { h.c. }
$$

Here and below implicit sums are over $1,2,3$ for $I, J, K$, over 1,2 , for $i, j, k$ and over 4,5 for $x, y$.

There is in fact no symmetry reason for the fields in $\chi_{L}$ to combine into a triplet [17]. More generally the coupling is of the form

$$
\mathcal{L}_{\text {top }}=-\lambda_{1} f \bar{\chi}_{L i} \epsilon^{i j} \epsilon^{x y} \Sigma_{j x} \Sigma_{3 y} q_{R}-\frac{1}{2} \lambda_{1}^{\prime} f \bar{u}_{L} \epsilon^{j k} \epsilon^{x y} \Sigma_{j x} \Sigma_{k y} q_{R}-\lambda_{2} f \bar{u}_{L} u_{R}+\text { h.c. }
$$

In this case, there is a quadratically divergent correction to the Higgs mass,

$$
\delta m_{h}^{2}=\frac{12}{16 \pi^{2}}\left(\lambda_{1}^{2}-\lambda_{1}^{\prime 2}\right) \Lambda^{2}
$$

where $\Lambda$ is a UV cut-off. As we will show below the relation $\lambda_{1}^{\prime}=\lambda_{1}$ is unstable against radiative corrections. For $f \approx 1 \mathrm{TeV}$ and $m_{h} \approx 100 \mathrm{GeV}$ this requires a tuning $\delta \lambda_{1}<0.04 \%$. Even if some unknown mechanism enforced $\lambda_{1}=\lambda_{1}^{\prime}$ at the cut-off $\Lambda$, running gives $\delta \lambda_{1}$ of order a few per cent at the scale of the Higgs mass; see eq. (4.34). If $\lambda_{1}(\Lambda)=\lambda_{1}^{\prime}(\Lambda)$ the correction to the Higgs mass is formally a two loop effect. However it is enhanced relative to the naive expectation by the large $\ln \left(4 \pi f / m_{h}\right) \approx 5$ and and the numerical factor of 12 in eq. (2.9). 


\section{$3 \quad$ General structure of counterterms}

In this section we study the structure of counterterms induced at 1-loop order in the $\mathrm{L}^{2} \mathrm{H}$ model (eqs. (2.5) and (2.7)). We focus on counterterms which have been neglected in the literature. We omit any discussion of non-derivative counterterms formed of scalar fields only, since the scalars' effective potential has been studied extensively, ${ }^{2}$ starting already with the original LLH paper [1].

\subsection{Scalar kinetic energy counterterms}

Kinetic energy counterterms are normally introduced in field theory by rescaling the bare fields $\phi \rightarrow Z^{1 / 2} \phi$. In non-linear sigma models the self-interactions of Goldstone bosons require counterterms that are higher order in the derivative expansion, and no rescaling of fields is necessary. However, non-linear sigma models coupled to light gauge bosons and fermions do generally require counterterms quadratic in derivatives. We will see that in the $\mathrm{L}^{2} \mathrm{H}$ model no rescaling $\phi \rightarrow Z^{1 / 2} \phi$ is needed. Instead new terms that are not symmetric under the full SU(5) symmetry are required to completely subtract the model at one loop.

We begin our study of the structure of kinetic energy counterterms by considering the slightly simpler case $\lambda_{1}^{\prime}=\lambda_{1}$. Working only to 1 loop, there is only one coupling constant present in each divergent self-energy diagram so the corresponding counterterm could just as well be computed setting all other coupling constants to zero. The Lagrangian with all but one couplings set to zero has an $\mathrm{SU}(3) \times \mathrm{SU}(2) \times \mathrm{U}(1)$ symmetry. Since we can choose the regulator to respect this symmetry we demand the counterterms are invariant under $\mathrm{SU}(3) \times \mathrm{SU}(2) \times \mathrm{U}(1)$.

Consider the possibility of partly subtracting the divergent graphs by rescaling the bare fields. In general we can choose a different wavefunction renormalization factor $Z$ for each of the fourteen Goldstone boson fields in $\Pi$. Were the interaction and the regularization method to respect the full flavor symmetry $(\mathrm{SU}(5))$, there would only be one common $Z$ for all the fields in $\Pi$. The question becomes: what is the restriction that $\mathrm{SU}(3) \times \mathrm{SU}(2) \times \mathrm{U}(1)$ imposes on the $Z$ ?

To answer this consider the expansion of the bare kinetic term

$$
\frac{f^{2}}{8} \operatorname{Tr} \partial_{\mu} \Sigma^{\dagger} \partial^{\mu} \Sigma=\operatorname{Tr} \partial_{\mu} \phi^{\dagger} \partial^{\mu} \phi+\frac{1}{2} \partial_{\mu} \eta \partial^{\mu} \eta+\operatorname{Tr} \partial_{\mu} \omega \partial^{\mu} \omega+\partial_{\mu} h^{\dagger} \partial^{\mu} h+\ldots
$$

where the ellipsis stand for terms quartic in the fields. Now we rescale each of the fourteen fields by an independent factor $Z$ and ask what are the constraints from imposing $\mathrm{SU}(3) \times$ $\mathrm{SU}(2) \times \mathrm{U}(1)$. There is a $\mathrm{SU}(2) \times \mathrm{U}(1)$ subgroup that acts linearly and hence there are only four different $Z$ factors:

$$
Z_{\phi} \operatorname{Tr} \partial_{\mu} \phi^{\dagger} \partial^{\mu} \phi+\frac{1}{2} Z_{\eta} \partial_{\mu} \eta \partial^{\mu} \eta+Z_{\omega} \operatorname{Tr} \partial_{\mu} \omega \partial^{\mu} \omega+Z_{h} \partial_{\mu} h^{\dagger} \partial^{\mu} h+\ldots
$$

We are led to consider the restrictions from $\mathrm{SU}(3)$ on these four factors. It is a straightforward but laborious exercise to compute the transformation properties of the fields in $\Pi$

\footnotetext{
${ }^{2}$ See ref. [27] for a detailed study of the 1-loop scalars' effective potential.
} 
under $\mathrm{SU}(3)$. We take for definiteness the $\mathrm{SU}(3)$ generated by the top-left $3 \times 3$ block. Of particular interest are transformations generated by the 4-7 Gell-Mann matrices

$$
\sum_{a=4}^{7} \epsilon^{a} T^{a}=\sum_{a=4}^{7} \epsilon^{a}\left(\begin{array}{cc}
\lambda^{a} & 0_{3 \times 2} \\
0_{2 \times 3} & 0_{2 \times 2}
\end{array}\right) \equiv\left(\begin{array}{ccc}
0_{2 \times 2} & \lambda & 0_{2 \times 2} \\
\lambda^{\dagger} & 0 & 0_{1 \times 2} \\
0_{2 \times 2} & 0_{2 \times 1} & 0_{2 \times 2}
\end{array}\right)
$$

where $\lambda$ is a $2 \times 1$ complex matrix of order $\epsilon$. The resulting nonlinear transformations, to first order in $\epsilon$, are

$$
\begin{aligned}
\delta h & =\frac{1}{\sqrt{2}} f \lambda+\frac{i}{\sqrt{2}}\left[-\omega \lambda-\frac{5}{\sqrt{20}} \eta \lambda+\phi \lambda^{*}\right]+\cdots \\
\delta \phi & =\frac{i}{2 \sqrt{2}}\left[h \lambda^{T}+\lambda h^{T}\right]+\cdots \\
\delta \eta & =i \frac{\sqrt{10}}{4}\left[h^{\dagger} \lambda-\lambda^{\dagger} h\right]+\cdots \\
\delta \omega & =\frac{i}{2 \sqrt{2}}\left[\lambda h^{\dagger}-h \lambda^{\dagger}\right]-\frac{i}{4 \sqrt{2}}\left[h^{\dagger} \lambda-\lambda^{\dagger} h\right] \mathbf{1}+\cdots
\end{aligned}
$$

where the ellipses stand for terms of quadratic and higher order in the fields.

Applying this variation to the kinetic term in (3.2) and retaining only terms quadratic in the fields we obtain

$$
\begin{aligned}
\delta \mathcal{L}= & \frac{1}{\sqrt{2}}\left(Z_{\phi}-Z_{h}\right) \operatorname{Tr} \partial_{\mu} \phi^{\dagger} \partial^{\mu} h \lambda^{T}+\text { h.c. } \\
& +\frac{1}{\sqrt{2}}\left(Z_{\omega}-Z_{h}\right) \operatorname{Tr} \partial_{\mu} \omega \partial^{\mu}\left[\lambda h^{\dagger}-h \lambda^{\dagger}\right]+\frac{\sqrt{10}}{4}\left(Z_{\eta}-Z_{h}\right) \operatorname{Tr} \partial_{\mu} \eta \partial^{\mu}\left[h^{\dagger} \lambda-\lambda^{\dagger} h\right] .
\end{aligned}
$$

Hence invariance under $\mathrm{SU}(3)$ requires $Z_{h}=Z_{\phi}=Z_{\omega}=Z_{\eta} \equiv Z$. The same conclusion is reached by consideration of other embeddings of the invariance subgroup.

Already in the special $\mathrm{SU}(3) \times \mathrm{SU}(2) \times \mathrm{U}(1)$-symmetric case one sees that divergences in the self-energy diagrams cannot be subtracted with a single common $Z$ factor. One must introduce counterterms invariant under $\mathrm{SU}(3) \times \mathrm{SU}(2) \times \mathrm{U}(1)$, or more generally, under $G_{w}$, that are not invariant under SU(5). We next turn to constructing the relevant counterterms.

\subsubsection{Scalar kinetic counterterms from gauge interaction}

Gauge interactions induce divergences in the scalar 2-point function with arbitrary background pion fields as shown in figure 1. We obtain the counterterms by the method of spurions. The gauge generators are promoted to spurions transforming in the adjoint representation of $\mathrm{SU}(5), T^{a} \rightarrow U T^{a} U^{\dagger}$. We list all the $\mathrm{SU}(5)$ invariant counterterms with two $T^{a}$ 's and two derivatives. In the $\mathrm{SU}(2)_{1}$ sector, with the generator $Q_{1}^{a}$ defined in eq. (2.1), 


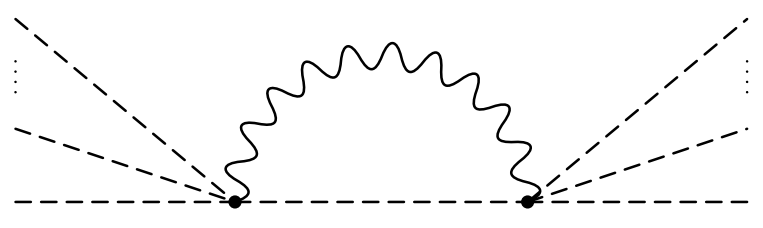

Figure 1. Scalar 2-point Function from gauge interaction with background pion fields.

we find

$$
\begin{aligned}
& \mathcal{O}_{1}^{g_{1}}=\operatorname{Tr}\left(Q_{1}^{a} Q_{1}^{a}\right) \operatorname{Tr}\left(D_{\mu} \Sigma D^{\mu} \Sigma^{*}\right), \\
& \mathcal{O}_{2}^{g_{1}}=\operatorname{Tr}\left(Q_{1}^{a} Q_{1}^{a} D_{\mu} \Sigma D^{\mu} \Sigma^{*}\right) \\
& \mathcal{O}_{3}^{g_{1}}=\operatorname{Tr}\left(Q_{1}^{a} D_{\mu} \Sigma\left(Q_{1}^{a}\right)^{T} D^{\mu} \Sigma^{*}\right) \\
& \mathcal{O}_{4}^{g_{1}}=\operatorname{Tr}\left(Q_{1}^{a}\left(D_{\mu} \Sigma\right) \Sigma^{*}\right) \operatorname{Tr}\left(Q_{1}^{a} \Sigma D^{\mu} \Sigma^{*}\right), \\
& \mathcal{O}_{5}^{g_{1}}=\operatorname{Tr}\left(Q_{1}^{a}\left(D_{\mu} \Sigma\right) \Sigma^{*} Q_{1}^{a} \Sigma D^{\mu} \Sigma^{*}\right), \\
& \mathcal{O}_{6}^{g_{1}}=\operatorname{Tr}\left(Q_{1}^{a} \Sigma\left(Q_{1}^{a}\right)^{T} \Sigma^{*}\right) \operatorname{Tr}\left(D_{\mu} \Sigma D^{\mu} \Sigma^{*}\right), \\
& \mathcal{O}_{7}^{g_{1}}=\operatorname{Tr}\left(Q_{1}^{a}\left(D_{\mu} \Sigma\right)\left(D^{\mu} \Sigma^{*}\right) \Sigma\left(Q_{1}^{a}\right)^{T} \Sigma^{*}\right)+\text { h.c.. }
\end{aligned}
$$

The counterterms for the $\mathrm{SU}(2)_{2}$ sector are obtained from those in the $\mathrm{SU}(2)_{1}$ sector by the replacements $g_{1} \rightarrow g_{2}$ and $Q_{1}^{a} \rightarrow Q_{2}^{a}$. For the $\mathrm{U}(1)_{1}$ sector, with the generator $Y_{1}$ defined in eq. (2.1), we have

$$
\begin{aligned}
& \mathcal{O}_{1}^{g_{1}^{\prime}}=\operatorname{Tr}\left(Y_{1} Y_{1}\right) \operatorname{Tr}\left(D_{\mu} \Sigma D^{\mu} \Sigma^{*}\right) \\
& \mathcal{O}_{2}^{g_{1}^{\prime}}=\operatorname{Tr}\left(Y_{1} Y_{1} D_{\mu} \Sigma D^{\mu} \Sigma^{*}\right) \\
& \mathcal{O}_{3}^{g_{1}^{\prime}}=\operatorname{Tr}\left(Y_{1} D_{\mu} \Sigma Y_{1} D^{\mu} \Sigma^{*}\right) \\
& \mathcal{O}_{4}^{g_{1}^{\prime}}=\operatorname{Tr}\left(Y_{1}\left(D_{\mu} \Sigma\right) \Sigma^{*}\right) \operatorname{Tr}\left(Y_{1} \Sigma D^{\mu} \Sigma^{*}\right) \\
& \mathcal{O}_{5}^{g_{1}^{\prime}}=\operatorname{Tr}\left(Y_{1}\left(D_{\mu} \Sigma\right) \Sigma^{*} Y_{1} \Sigma D^{\mu} \Sigma^{*}\right) \\
& \mathcal{O}_{6}^{g_{1}^{\prime}}=\operatorname{Tr}\left(Y_{1} \Sigma Y_{1} \Sigma^{*}\right) \operatorname{Tr}\left(D_{\mu} \Sigma D^{\mu} \Sigma^{*}\right) \\
& \mathcal{O}_{7}^{g_{1}^{\prime}}=\operatorname{Tr}\left(Y_{1}\left(D_{\mu} \Sigma\right)\left(D^{\mu} \Sigma^{*}\right) \Sigma Y_{1} \Sigma^{*}\right)+\text { h.c.. }
\end{aligned}
$$

Similarly, the counterterms for the $\mathrm{U}(1)_{2}$ sector can be obtained by substituting $g_{1}^{\prime} \rightarrow g_{2}^{\prime}$ and $Y_{1} \rightarrow Y_{2}$ in the operators above.

\subsubsection{Scalar kinetic counterterms from Yukawa interaction}

Yukawa interactions also induce divergences in the scalar 2-point function with arbitrary background pion fields as shown in figure 2. Just as was done for gauge generators, we treat the Yukawa couplings as $\mathrm{SU}(5)$ breaking spurions. In doing so, we promote $\chi_{L}$ to a 5-plet

$$
\mathcal{L}_{Y u k}=\bar{\chi}_{L a} S^{a b c d e} \Sigma_{b c} \Sigma_{d e} q_{R}+\text { h.c. }
$$




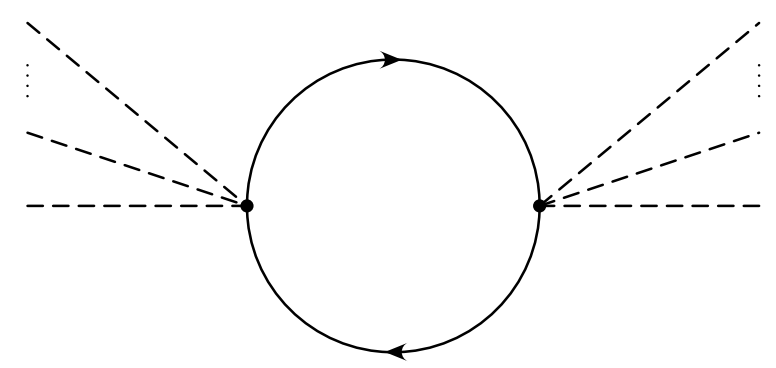

Figure 2. Scalar 2-point Function from Yukawa interaction with background pion fields.

with $S$ symmetric in $\{b, c\},\{d, e\}$ and the exchange of the pair $(b, c) \leftrightarrow(d, e)$. The spurion $S$ is not arbitrary, but rather takes a fixed "vacuum expectation" value

$$
<S^{a b c d e}>= \begin{cases}\frac{\lambda_{1}}{8} \epsilon^{a b d 45} \epsilon^{123 c e}+\ldots & a=1,2 \\ \frac{\lambda_{1}^{\prime}}{8} \epsilon^{3 b d 45} \epsilon^{123 c e}+\ldots & a=3\end{cases}
$$

where $+\ldots$ stands for symmetrization. Note that we can demand that $S \rightarrow S^{*}$ under CP, so $\mathcal{L}$ is invariant under $\mathrm{CP}$. The counterterms will be also invariant under $\mathrm{CP}$ and hence hermitian. For notational compactness we define $\Psi^{a}=S^{a b c d e} \Sigma_{b c} \Sigma_{d e}$. In terms of this, the counterterm is

$$
\mathcal{O}_{\Psi}=D_{\mu} \Psi^{\dagger a} D^{\mu} \Psi_{a}
$$

\subsection{Fermion kinetic energy counterterms}

The divergence in the fermion self-energy is also present in the diagram with arbitrary number of pion fields at each of the Yukawa vertices, as shown in figure 3. For notational compactness we defined $\Psi^{a b c}=S^{a b c d e} \Sigma_{d e}$ and $\xi_{a b c}=S_{a b c d e}^{*} \Sigma^{* d e}$. The counterterms for the $q_{R} 2$-point function are

$$
\begin{aligned}
\mathcal{O}_{q_{1}} & =\bar{q}_{R} \bar{\Psi}_{a b c} i \not D \Psi^{a b c} q_{R}, \\
\mathcal{O}_{q_{2}} & =\bar{q}_{R} \bar{\Psi}_{a b c} \Sigma^{* c e} i \not D \Sigma_{e d} \Psi^{a b d} q_{R}, \\
\mathcal{O}_{q_{3}} & =\bar{q}_{R} \bar{\Psi}_{a b c} \Sigma^{* b c} i \not D \Sigma_{d e} \Psi^{a d e} q_{R}, \\
\mathcal{O}_{q_{4}} & =\bar{q}_{R} \bar{\Psi}_{a b c} \Sigma_{d e} i \not D \Sigma^{* b c} \Psi^{a d e} q_{R}, \\
\mathcal{O}_{q_{5}} & =\bar{q}_{R} \bar{\Psi}_{a b c} \gamma^{\mu} \Psi^{a d e} D_{\mu}\left(\Sigma^{* b c} \Sigma_{d e}\right) q_{R},
\end{aligned}
$$

while the counterterms for $\chi_{L}$ 2-ponit function are

$$
\begin{aligned}
& \mathcal{O}_{\chi_{1}}=\bar{\chi}_{L a} \bar{\xi}^{a b c} i \not D \xi_{a^{\prime} b c} \chi_{L}^{a^{\prime}} \\
& \mathcal{O}_{\chi_{2}}=\bar{\chi}_{L a} \bar{\xi}^{a b c} \Sigma_{c e} i \not D \Sigma^{* e d} \xi_{a^{\prime} b d} \chi_{L}^{a^{\prime}}, \\
& \mathcal{O}_{\chi_{3}}=\bar{\chi}_{L a} \bar{\xi}^{a b c} \Sigma_{b c} i \not D \Sigma^{* d e} \xi_{a^{\prime} d e} \chi_{L}^{a^{\prime}} \\
& \mathcal{O}_{\chi_{4}}=\bar{\chi}_{L a} \bar{\xi}^{a b c} \Sigma^{* d e} i \not D \Sigma_{b c} \xi_{a^{\prime} d e} \chi_{L}^{a^{\prime}} \\
& \mathcal{O}_{\chi_{5}}=\bar{\chi}_{L a} \bar{\xi}^{a b c} \gamma^{\mu} \xi_{a^{\prime} d e} D_{\mu}\left(\Sigma^{* d e} \Sigma_{b c}\right) \chi_{L}^{a^{\prime}}
\end{aligned}
$$




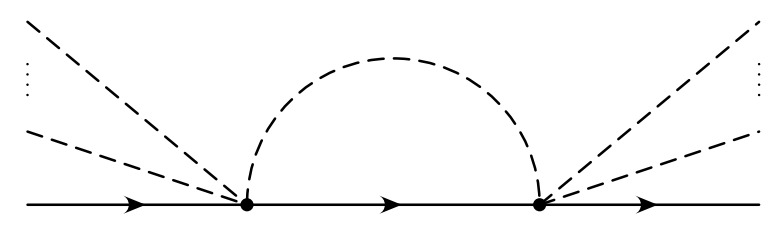

Figure 3. Fermion 2pt Function from Yukawa interaction with background pion fields.

\subsection{Yukawa vertex counterterms}

At 1-loop order, gauge interactions do not introduce a new counterterm. So we can subtract off the divergences with the Yukawa operator (i.e., $\bar{\chi}_{L a} S^{a b c d e} \Sigma_{b c} \Sigma_{d e} q_{R}+$ h.c.). This is not the case for Yukawa interactions which generate two new counterterms

$$
\begin{aligned}
& \mathcal{O}_{v_{1}}=\bar{q}_{R} S_{a b c d e}^{*} \Sigma^{* d e} S^{l m n o p} \Sigma_{m n} \Sigma_{o p} S_{l q r s t}^{*} \Sigma^{* s t} \Sigma^{* b c} \Sigma^{* q r} \chi_{L}^{a}+\text { h.c. }, \\
& \mathcal{O}_{v_{2}}=\bar{q}_{R} S_{a b c d e}^{*} \Sigma^{* d e} S^{l m n o p} \Sigma_{m n} \Sigma_{o p} S_{l q r s t}^{*} \Sigma^{* s t} \Sigma^{* b q} \Sigma^{* c r} \chi_{L}^{a}+\text { h.c. }
\end{aligned}
$$

\subsection{Counterterms to counterterms: the general case}

The counterterms displayed so far are appropriate to render all green functions finite if the only interactions in the model are those displayed in the Lagrangian given in section 2 . That is, the counterterms are appropriate to the case were the bare Lagrangian has the form given in section 2. However, this cannot be maintained beyond 1-loop order. The 1-loop counterterms become interaction terms at 2-loops. This requires additional counterterms. And so on, as one moves to higher orders in the loop expansion. All terms consistent with the symmetries of the model will be generated by renormalization.

It is more natural to start with the complete set of interaction terms (formerly counterterms) and treat them all on an equal footing. However, this is not a viable program for this model since the complete set does not appear to be finite. The next best option is an organizing principle for a calculation that requires finite precision.

Before we make a specific proposal for one such organizing principle, we would like to contrast this with other models. Clearly the case of renormalizable theories is very different: only a finite number of terms is required to renormalize the theory to all orders in the loop expansion. More apropos, the case of chiral Lagrangians is different too. For these as one goes up in the loop expansion the counterterms involve accordingly more derivatives. Therefore the infinite set of counterterms are neatly organized by the number of derivatives which is tied to the loop expansion. In the $\mathrm{L}^{2} \mathrm{H}$ this is explicitly not the case already at 1-loop order: the counterterms generated are not suppressed by additional derivatives.

Suppose we are interested in processes that do not involve more than $n$ PGBs. By expanding the $\Sigma$ field in powers of the PGBs we will discover there is a finite number, $N(n, d)$ of linearly independent operators containing no more than $d$ derivatives. Denote this basis of operators by $\widehat{\mathcal{O}}_{i}$. Then one can re-define the remaining (infinite set of) operators so that their expansion in PGBs starts at order higher than $n, \mathcal{O}_{a} \rightarrow \mathcal{O}_{a}-\sum_{i} c_{a}^{i} \widehat{\mathcal{O}}_{i}$ where the sum runs to $N(n, d)$. Given a desired precision for a calculation one can determine the order in the loop and momentum expansions required to achieve that precision. The latter gives 
us directly the required number of derivatives $d$ to be retained. The number $n$ of PGBs to be retained is a bit more complicated. For a process that involves $k$ PGBs, an operator with $k+2 L$ PGBs can contribute at $L$-loop order. Therefore, for processes with no more than $k$ PGBs that require $L$-loop precision and up to $d$ powers of momenta, the basis with $N(k+2 L, d)$ operators should be used.

While the above algorithm is quite specific, we have not carried out that program of renormalization. The reason should be clear: the algorithm requires making a specific choice of process to study, or at least a restriction on the number of PGBs in the processes that will be considered. So, as explained at the top of this section, we have opted instead for the full 1-loop renormalization of the model of section 2 assuming all other possible terms consistent with symmetries (an infinite set) is absent in the bare Lagrangian.

\section{Renormalization}

\subsection{Generalities}

The renormalized Lagrangian is

$$
\mathcal{L}=\mathcal{L}_{\phi}+\mathcal{L}_{\psi}+\mathcal{L}_{\text {Yuk }}
$$

where

$$
\begin{aligned}
\mathcal{L}_{\phi}= & \frac{f^{2}}{8}\left[\operatorname{Tr}\left(D_{\mu} \Sigma^{\dagger} D^{\mu} \Sigma\right)+\sum_{a, i} \zeta_{a}^{g_{i}} Z_{a}^{g_{i}} \mathcal{O}_{a}^{g_{i}}+\mu^{-\epsilon} \kappa_{\Psi} Z_{\Psi} \mathcal{O}_{\Psi}\right] \\
\mathcal{L}_{\psi}= & Z_{\chi_{L}} \bar{\chi}_{L I} i \not D \chi_{L}^{I}+Z_{q_{R}} \bar{q}_{R} i \not D q_{R} \\
& +\mu^{-\epsilon} \sum_{a=1}^{5} \kappa_{\chi_{a}} Z_{\chi_{a}} Z_{\chi_{L}} \mathcal{O}_{\chi_{a}}+\mu^{-\epsilon} \sum_{b=1}^{5} \kappa_{q_{b}} Z_{q_{b}} Z_{q_{R}} \mathcal{O}_{q_{b}}, \\
\mathcal{L}_{\text {Yuk }}= & -f \mu^{\epsilon / 2} \lambda_{1} Z_{\lambda}\left(Z_{\chi_{L}} Z_{q_{R}}\right)^{1 / 2} \bar{\chi}_{L i} \epsilon^{i j} \epsilon^{x y} \Sigma_{j x} \Sigma_{3 y} q_{R}+\text { h.c. } \\
& -\frac{f}{2} \mu^{\epsilon / 2} \lambda_{1}^{\prime} Z_{\lambda^{\prime}}\left(Z_{\chi_{L}} Z_{q_{R}}\right)^{1 / 2} \bar{u}_{L} \epsilon^{j k} \epsilon^{x y} \Sigma_{j x} \Sigma_{k y} q_{R}+\text { h.c. } \\
& +f \mu^{-\epsilon} \sum_{a=1}^{2} \kappa_{v_{a}} Z_{v_{a}} \mathcal{O}_{v_{a}} .
\end{aligned}
$$

and the wavefunction renormalization of the Goldstone bosons is implicit in $\Sigma=\exp \left(2 i Z^{1 / 2} \Pi / f\right) \Sigma_{0}$. Note that we have kept the bare $f$ throughout, and it has dimension $1-\epsilon / 2$ in dimensional regularization (with $d=4-\epsilon$ ). This ensures that the coefficients of the power expansion of the kinetic terms do not run (or rather, they all run the same, just according to the wavefunction of the field $\Pi$ ). Since the spurion $S^{\text {abcde }}$ includes the Yukawa coupling constants it has dimension $\epsilon / 2$. Therefore, the bare couplings $\kappa$ have dimension $-\epsilon$. We have ignored the $\lambda_{2}$ term in the Yukawa Lagrangian as it plays no role in renormalization.

The calculation will require we fix the U(1) charges of all fields. For the $\Sigma$ fields these are already determined by the transformation properties under $G_{f}$, and the fact that $G_{w}$ is a subgroup of $G_{f}$. Since the hypercharges $Y=Y_{1}+Y_{2}$ are fixed and the interactions 
are invariant under the gauge transformations, there is only freedom to choose the $\mathrm{U}(1)$ charge of one quark field. We take $q_{R}$ to have $Y_{2}$ charge $y$. Then the rest of the charges are fixed:

$$
\begin{array}{rlrl}
Y_{1}\left(q_{R}\right) & =2 / 3-y & & Y_{2}\left(q_{R}\right)=y \\
Y_{1}(\chi)=\frac{11}{30}-y & & Y_{2}\left(\chi_{L}\right)=y-\frac{1}{5} \\
Y_{1}\left(u_{L}\right) & =\frac{13}{15}-y & & Y_{2}\left(u_{L}\right)=y-\frac{1}{5}
\end{array}
$$

The $\zeta_{a}$ and $\kappa_{a}$ terms modify the Lagrangian at tree level and these modifications should be included in our perturbative computations. However, we intend to take the bare parameters $\zeta_{a}$ and $\kappa_{a}$ to vanish at the end of the calculation. This is because we want to study the radiative corrections that generate these terms, even if absent from the bare Lagrangian. Then, while $\zeta_{a}$ terms in the tree level Feynman rules can be neglected, the counterterms, of the form $\zeta_{a}\left(Z_{a}-1\right)$, do not vanish as $\zeta_{a} \rightarrow 0$ (similarly for $\kappa_{a}$ terms). The RGE for these couplings is derived through standard methods. We use a generic coupling $\zeta$ for the couplings of $\zeta, \kappa$ terms. Taking a log-derivative with respect to $\mu$ of $\zeta^{\text {bare }}=\mu^{\epsilon D_{\zeta}} Z \zeta$, where $\epsilon D_{\zeta}$ is the dimension of the bare coupling $\zeta$, we have

$$
\epsilon D_{\zeta} Z \zeta+\mu \frac{\partial \zeta}{\partial \mu}\left(Z+\zeta \frac{\partial Z}{\partial \zeta}\right)+\zeta\left(-\frac{1}{2} \epsilon g+\beta_{g}\right) \frac{\partial Z_{a}}{\partial g}=0 .
$$

Here $g$ stands for the collection of Yukawa and gauge coupling constants, and there is an implicit sum over these. Since $\mu \frac{\partial \zeta}{\partial \mu}$ has a finite limit as $\epsilon \rightarrow 0$ and $Z$ can be written as

$$
Z=1+\frac{a^{(1)}}{\zeta} \frac{1}{\epsilon}+O\left(\epsilon^{-2}\right)
$$

where $a^{(1)}=a^{(1)}(g)$ is only a function of the couplings, we have

$$
\begin{aligned}
\mu \frac{\partial \zeta}{\partial \mu} & =-\epsilon D_{\zeta} \zeta+\beta_{\zeta}, \\
\beta_{\zeta} & =-D_{\zeta} a^{(1)}+\frac{1}{2} g \frac{\partial a^{(1)}}{\partial g} .
\end{aligned}
$$

We will determine the normalization factors $Z_{a}$ in the next subsection.

\subsection{Matching counterterms}

\subsubsection{Scalar 2-Point functions with arbitrary scalar background}

We first consider the $\mathrm{SU}(2)_{1}$ gauge sector. The non-trivial 2-point functions for the scalars are

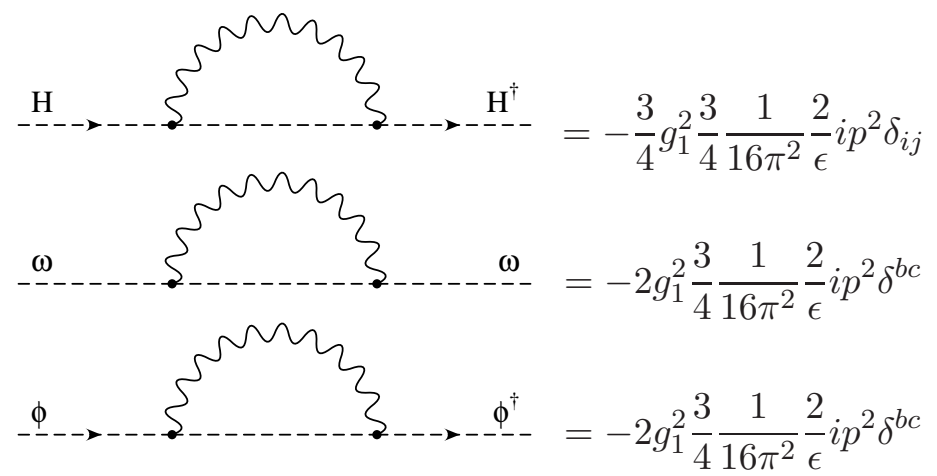


There is no $\eta$ self-enrgy diagram because it is a singlet under the gauge group. For the 3 -point functions, we denote by $p_{i}$ the momentum of particle $i$ starting from the left in the clockwise direction in the following diagrams
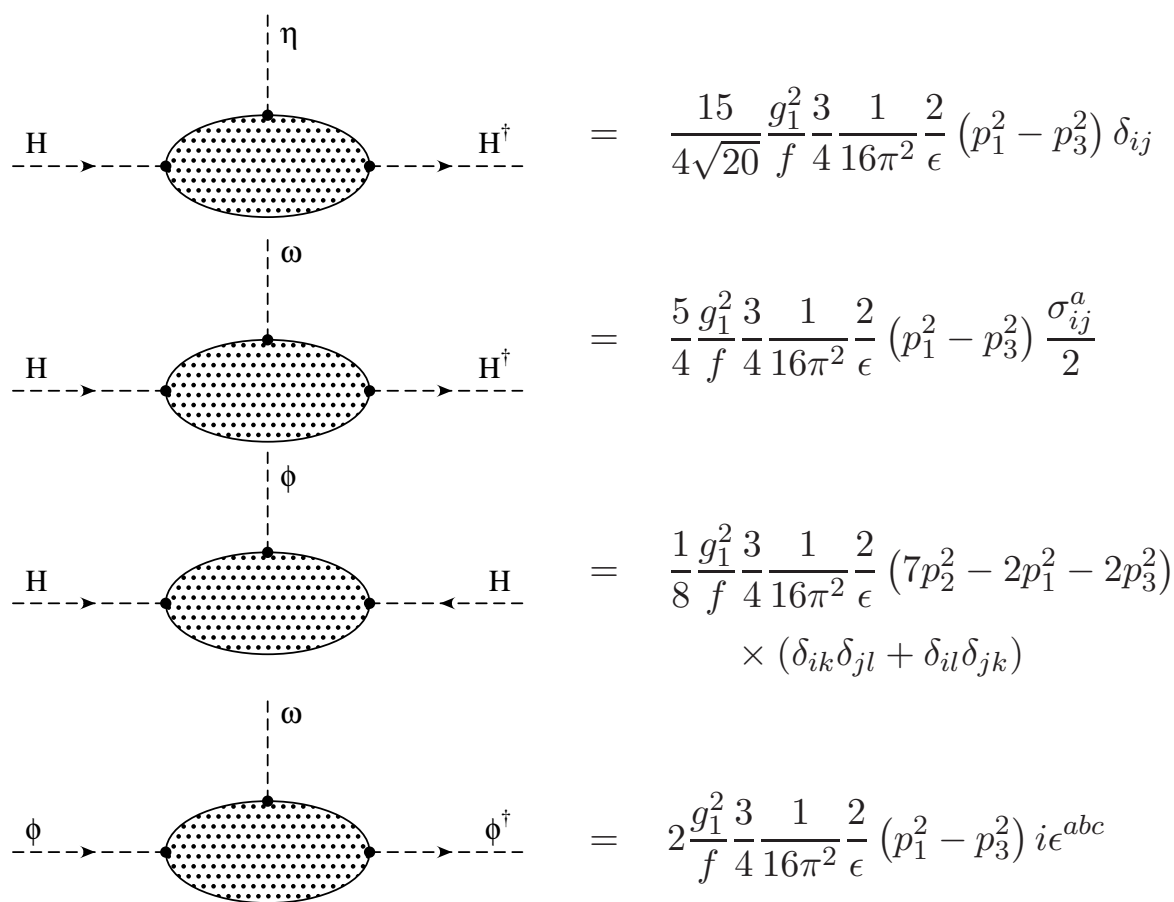

where we used $\omega=\omega^{a} \sigma^{a} / 2$ and $\phi=\sigma^{a} \sigma^{2} \phi^{a} / \sqrt{2}$. We have also found that the 4-point function with two $\eta$ 's and two $\phi$ 's vanishes. Cancelation of divergences in these diagrams, together with the absence of diagram with two $\eta$ 's and two $\phi$ 's requires

$$
\begin{aligned}
Z & =1, & Z_{4}^{g_{1}} & =1, \\
Z_{1}^{g_{1}} & =1, & Z_{5}^{g_{1}} & =1-3 \frac{1}{\zeta_{5}^{g_{1}}} \frac{g_{1}^{2}}{16 \pi^{2}} \frac{2}{\epsilon}, \\
Z_{2}^{g_{1}} & =1+3 \frac{1}{\zeta_{2}^{g_{1}} \frac{g_{1}^{2}}{16 \pi^{2}} \frac{2}{\epsilon},} & Z_{6}^{g_{1}} & =1+\frac{3}{20} \frac{1}{\zeta_{6}^{g_{1}}} \frac{g_{1}^{2}}{16 \pi^{2}} \frac{2}{\epsilon}, \\
Z_{3}^{g_{1}} & =1+3 \frac{1}{\zeta_{3}^{g_{1}}} \frac{g_{1}^{2}}{16 \pi^{2}} \frac{2}{\epsilon}, & Z_{7}^{g_{1}} & =1-\frac{3}{2} \frac{1}{\zeta_{7}^{g_{1}}} \frac{g_{1}^{2}}{16 \pi^{2}} \frac{2}{\epsilon},
\end{aligned}
$$

We can similarly determine $Z_{i}^{g_{2}}$ by nothing that $\mathcal{L}_{g_{2}} \rightarrow \mathcal{L}_{g_{1}}$ and $\mathcal{O}_{i}^{g_{2}} \rightarrow \mathcal{O}_{i}^{g_{1}}$ when $\Pi \rightarrow-\Pi$. Thus we have $Z_{i}^{g_{1}}=Z_{i}^{g_{2}}$. 
We next consider the $\mathrm{U}(1)_{1}$ sector. The divergent 2-point and 3-point functions are
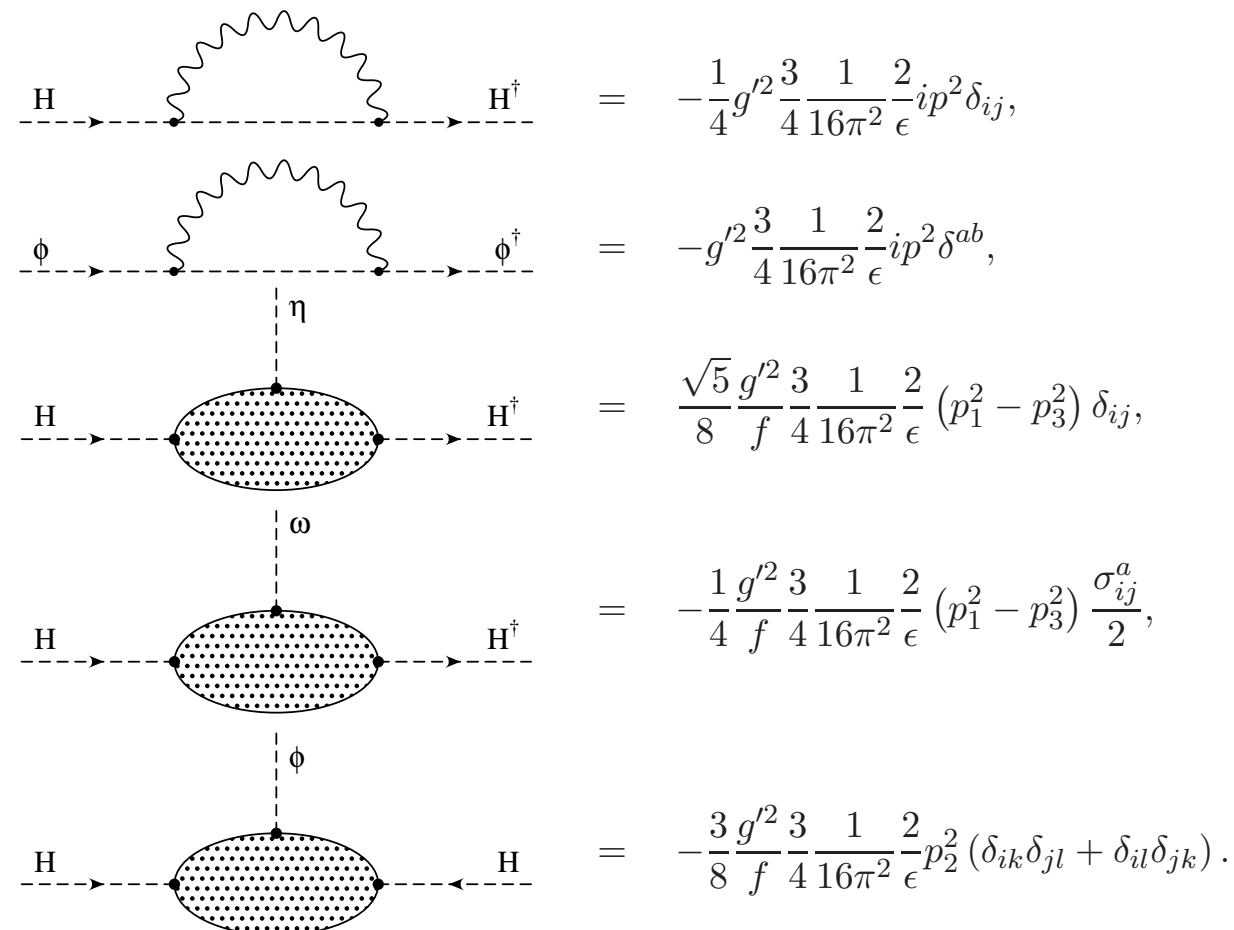

As in the case of $\mathrm{SU}(2)$, the 4-point function with two $\eta$ 's and two $\phi$ 's vanishes. Cancelation of divergences in these diagrams, together with the absence of a divergence in the diagram with two $\eta \mathrm{s}$ and two $\phi \mathrm{s}$, requires

$$
\begin{array}{rlrl}
Z_{1}^{g_{1}^{\prime}} & =1-\frac{1}{40} \frac{1}{\zeta_{1}^{g_{1}^{\prime}}} \frac{g_{1}^{\prime 2}}{16 \pi^{2}} \frac{2}{\epsilon}, & Z_{4}^{g_{1}^{\prime}} & =1, \\
Z_{5}^{g_{1}^{\prime}} & =1-\frac{3}{8} \frac{1}{\zeta_{5}^{g_{1}}} \frac{g_{1}^{\prime 2}}{16 \pi^{2}} \frac{2}{\epsilon}, \\
Z_{2}^{g_{1}^{\prime}} & =1+\frac{3}{8} \frac{1}{\zeta_{2}^{g_{1}^{\prime}}} \frac{g_{1}^{\prime 2}}{16 \pi^{2}} \frac{2}{\epsilon}, & Z_{6}^{g_{1}^{\prime}} & =1-\frac{3}{80} \frac{1}{\zeta_{6}^{g_{1}^{\prime}}} \frac{g_{1}^{\prime 2}}{16 \pi^{2}} \frac{2}{\epsilon}, \\
Z_{3}^{g_{1}^{\prime}}=1+\frac{3}{8} \frac{1}{\zeta_{3}^{g_{1}^{\prime}}} \frac{g_{1}^{\prime 2}}{16 \pi^{2}} \frac{2}{\epsilon}, & Z_{7}^{g_{1}^{\prime}} & =1-\frac{3}{16} \frac{1}{\zeta_{7}^{g_{1}^{\prime}}} \frac{g_{1}^{\prime 2}}{16 \pi^{2}} \frac{2}{\epsilon},
\end{array}
$$

and $Z_{i}^{g_{1}^{\prime}}=Z_{i}^{g_{2}^{\prime}}$. The divergence in the $H$ 2-point function from Yukawa interaction is

$$
\frac{2 \lambda_{1}^{2}}{16 \pi^{2}} \frac{2}{\epsilon}
$$

and in the $\eta 2$-point function is

$$
\frac{8}{5} \frac{\lambda_{1}^{\prime 2}}{16 \pi^{2}} \frac{2}{\epsilon}
$$

Thus we obtain

$$
Z_{\Psi}=1-\frac{1}{\kappa_{\psi}} \frac{1}{16 \pi^{2}} \frac{2}{\epsilon}
$$




\subsubsection{Fermion 2-point functions}

We first consider the $q_{R}$ 2-point functions with arbitrary scalar background. The 1-loop diagrams are
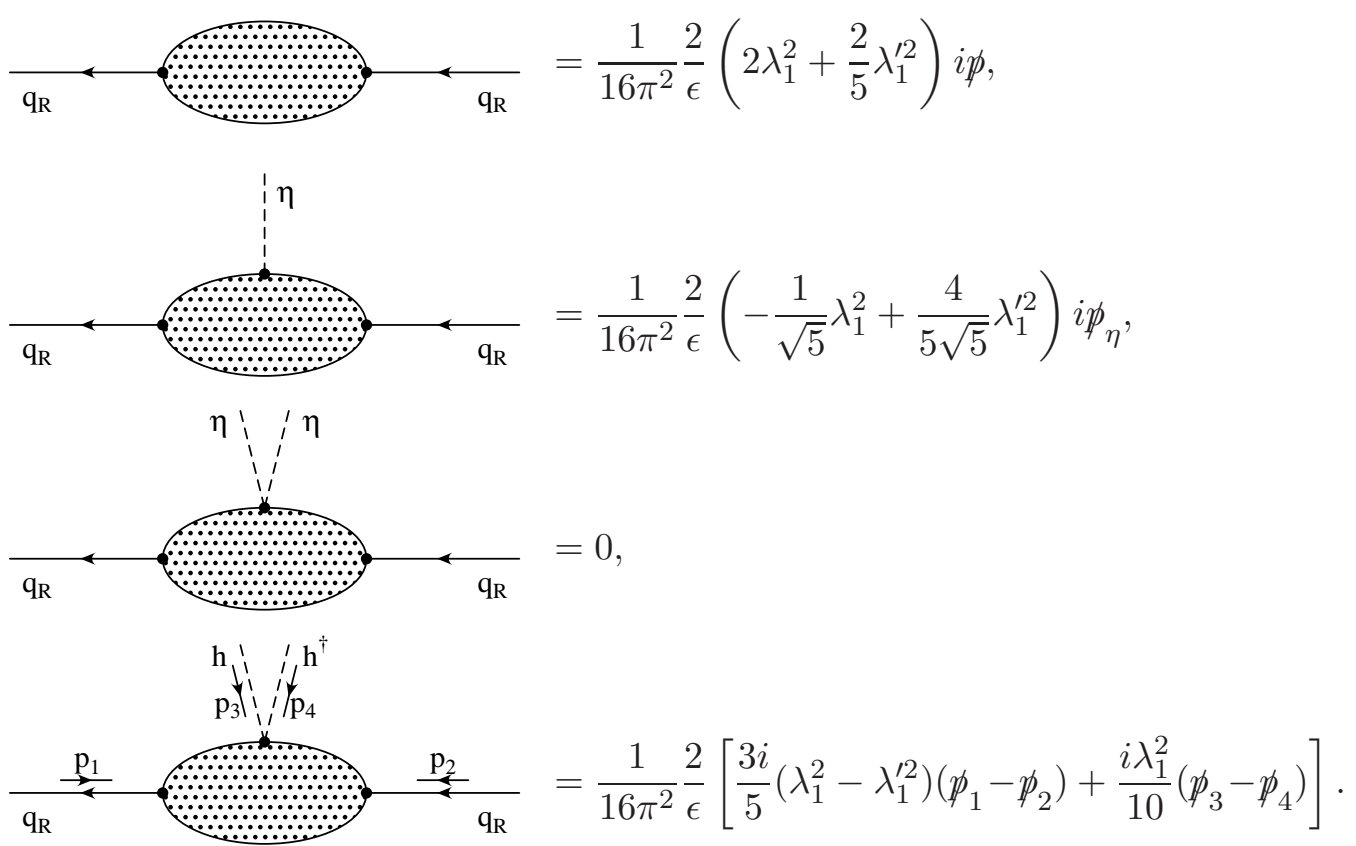

Matching counterterms yields

$$
\begin{array}{lll}
Z_{q_{R}}=1, & Z_{q_{2}}=1-8 \frac{1}{\kappa_{q_{2}}} \frac{1}{16 \pi^{2}} \frac{2}{\epsilon}, & Z_{q_{4}}=1, \\
Z_{q_{1}}=1, & Z_{q_{3}}=1+\frac{8}{5} \frac{1}{\kappa_{q_{3}}} \frac{1}{16 \pi^{2}} \frac{2}{\epsilon}, & Z_{q_{5}}=1 .
\end{array}
$$

Similarly, for the $\chi_{L}$ 2-point functions, we find

$$
\begin{aligned}
& Z_{\chi_{L}}=1, \quad Z_{\chi_{2}}=1-8 \frac{1}{\kappa_{\chi_{2}}} \frac{1}{16 \pi^{2}} \frac{2}{\epsilon}, \quad Z_{\chi_{4}}=1, \\
& Z_{\chi_{1}}=1, \quad Z_{\chi_{3}}=1+\frac{8}{5} \frac{1}{\kappa_{\chi_{3}}} \frac{1}{16 \pi^{2}} \frac{2}{\epsilon}, \quad Z_{\chi_{5}}=1 .
\end{aligned}
$$

\subsubsection{Yukawa vertex counterterms}

As we mentioned above, gauge interactions do not induce new operators but the Yukawa interaction do. Here we distinguish the $\mathrm{SU}(2)$ and $\mathrm{U}(1)$ part of the Yukawa in their action:

$$
\begin{aligned}
& Z_{\lambda_{1}}=1-3 \frac{1}{16 \pi^{2}} \frac{2}{\epsilon}\left[\left(\frac{11}{30}-y\right)\left(\frac{2}{3}-y\right) g_{1}^{\prime 2}+\left(y-\frac{1}{5}\right) y g_{2}^{\prime 2}\right], \\
& Z_{\lambda_{1}^{\prime}}=1-3 \frac{1}{16 \pi^{2}} \frac{2}{\epsilon}\left[\left(\frac{13}{15}-y\right)\left(\frac{2}{3}-y\right) g_{1}^{\prime 2}+\left(y-\frac{1}{5}\right) y g_{2}^{\prime 2}\right] .
\end{aligned}
$$


We see that $\lambda_{1}$ and $\lambda_{1}^{\prime}$ are renormalized differently. The other renomalization factors are:

$$
\begin{aligned}
& Z_{v_{1}}=1+\frac{4}{5} \frac{1}{\kappa_{v_{1}}} \frac{1}{16 \pi^{2}} \frac{2}{\epsilon}, \\
& Z_{v_{2}}=1+\frac{11}{5} \frac{1}{\kappa_{v_{2}}} \frac{1}{16 \pi^{2}} \frac{2}{\epsilon} .
\end{aligned}
$$

\section{$4.3 \beta$ functions}

We have already pointed out that the coupling $\lambda_{1}$ and $\lambda_{1}^{\prime}$ run differently [17]. It is now straightforward to obtain their beta functions:

$$
\begin{aligned}
& \frac{\beta_{\lambda_{1}}}{\lambda_{1}}=-\frac{3}{8 \pi^{2}}\left[\left(\frac{11}{30}-y\right)\left(\frac{2}{3}-y\right) g_{1}^{\prime 2}+\left(y-\frac{1}{5}\right) y g_{2}^{\prime 2}\right] \\
& \frac{\beta_{\lambda_{1}^{\prime}}}{\lambda_{1}^{\prime}}=-\frac{3}{8 \pi^{2}}\left[\left(\frac{13}{15}-y\right)\left(\frac{2}{3}-y\right) g_{1}^{\prime 2}+\left(y-\frac{1}{5}\right) y g_{2}^{\prime 2}\right]
\end{aligned}
$$

Note, in particular, that

$$
\mu \frac{\partial}{\partial \mu} \ln \left(\frac{\lambda_{1}}{\lambda_{1}^{\prime}}\right)=\left(\frac{2}{3}-y\right) \frac{3 g_{1}^{\prime 2}}{16 \pi^{2}}
$$

With $\beta_{g_{1}^{\prime}}=\left(b / 16 \pi^{2}\right) g_{1}^{\prime 3}$ we can write the solution in terms of the running coupling:

$$
\frac{\lambda_{1}(\mu)}{\lambda_{1}^{\prime}(\mu)}=\frac{\lambda_{1}(\Lambda)}{\lambda_{1}^{\prime}(\Lambda)}\left(\frac{g_{1}^{\prime}(\mu)}{g_{1}^{\prime}(\Lambda)}\right)^{\frac{2-3 y}{b}}
$$

The $\beta$ functions for the couplings $\zeta_{a}^{g}$ are determined using eq. (4.7). We find

$$
\begin{array}{lll}
\beta_{\zeta_{1}}^{g_{1(2)}}=0, & \beta_{\zeta_{1}}^{g_{1(2)}^{\prime}}=-\frac{1}{20} \frac{g_{1(2)}^{\prime 2}}{16 \pi^{2}}, \\
\beta_{\zeta_{2}}^{g_{1(2)}}=6 \frac{g_{1(2)}^{2}}{16 \pi^{2}}, & \beta_{\zeta_{2}}^{g_{1(2)}^{\prime}}=\frac{3}{4} \frac{g_{1(2)}^{\prime 2}}{16 \pi^{2}}, \\
\beta_{\zeta_{3}}^{g_{1(2)}}=6 \frac{g_{1(2)}^{2}}{16 \pi^{2}}, & \beta_{\zeta_{3}}^{g_{1(2)}^{\prime}}=\frac{3}{4} \frac{g_{1(2)}^{\prime 2}}{16 \pi^{2}}, \\
\beta_{\zeta_{4}}^{g_{1(2)}}=0, & \beta_{\zeta_{4}}^{g_{1(2)}^{\prime}}=0, \\
\beta_{\zeta_{5}}^{g_{1(2)}}=-6 \frac{g_{1(2)}^{2}}{16 \pi^{2}}, & \beta_{\zeta_{5}}^{g_{1(2)}^{\prime}}=-\frac{3}{4} \frac{g_{1(2)}^{\prime 2}}{16 \pi^{2}}, \\
\beta_{\zeta_{6}}^{g_{1(2)}}=\frac{3}{10} \frac{g_{1(2)}^{2}}{16 \pi^{2}}, & \beta_{\zeta_{6}}^{g_{1(2)}^{\prime}}=-\frac{3}{40} \frac{g_{1(2)}^{\prime 2}}{16 \pi^{2}}, \\
\beta_{\zeta_{7}}^{g_{1(2)}}=-3 \frac{g_{1(2)}^{2}}{16 \pi^{2}}, & \beta_{\zeta_{7}}^{g_{1(2)}^{\prime}}=-\frac{3}{8} \frac{g_{1(2)}^{\prime 2}}{16 \pi^{2}} .
\end{array}
$$

For $\kappa$ the couplings are implicit in the operators so the $\beta$ functions are pure number

$$
\begin{array}{lll}
\beta_{\kappa_{q_{1}}}=0, & \beta_{\kappa_{\chi_{1}}}=0, \\
\beta_{\kappa_{q_{2}}}=-16 \frac{1}{16 \pi^{2}}, & \beta_{\kappa_{\chi_{2}}}=-16 \frac{1}{16 \pi^{2}}, \\
\beta_{\kappa_{q_{3}}}=\frac{16}{5} \frac{1}{16 \pi^{2}}, & \beta_{\kappa_{\chi_{3}}}=+\frac{16}{5} \frac{1}{16 \pi^{2}}, \\
\beta_{\kappa_{q_{4}}}=0, & \beta_{\kappa_{\chi_{4}}}=0, \\
\beta_{\kappa_{q_{5}}}=0, & \beta_{\kappa_{\chi_{5}}}=0,
\end{array}
$$


and

$$
\beta_{\psi}=-2 \frac{1}{16 \pi^{2}}, \quad \beta_{\kappa_{v_{1}}}=\frac{8}{5} \frac{1}{16 \pi^{2}}, \quad \beta_{\kappa_{v_{2}}}=\frac{22}{5} \frac{1}{16 \pi^{2}}
$$

\section{Conclusions}

We have studied the one loop renormalization of the Littlest Higgs Model. Phenomenologically this model has fallen somewhat out of favor because of its difficulties simultaneously accommodating electroweak precision constraints and solving the little hierarchy problem [7-11]. However, its structure is prototypical of many models, like Littlest Higgs models with reduced gauge symmetry [20], or with custodial [12, 13] or T-parity [14-16] symmetries. Therefore, the methods introduced here should be largely the same as those needed for one loop renormalization of any model in this class.

We have displayed explicit counterterms and their $Z$ factors in dimensional regularization, in Landau gauge. These results are only of interest to understand the procedure, so they have been included here more for clarity of presentation. However, the beta functions of the couplings of all the terms in the Lagrangian are independent of gauge and scheme choice. They, together with the methods introduced, constitute the main result of this work and are displayed explicitly in section 4 .

One important result is that the coupling constants associated with the Yukawa coupling of the top quark run differently; see eq. (4.33). As observed in ref. [17] in the absence of fine tuning, the collective symmetry mechanism fails for Yukawa couplings in the Littlest Higgs model and its relatives. One can similarly conclude that the terms that were required as counterterms, all allowed by the symmetries and being of leading order in the derivative expansion, should have been included in the model from the start.

\section{Acknowledgments}

Work supported in part by the US Department of Energy under contract DE-FG0397ER40546.

Open Access. This article is distributed under the terms of the Creative Commons Attribution Noncommercial License which permits any noncommercial use, distribution, and reproduction in any medium, provided the original author(s) and source are credited.

\section{References}

[1] N. Arkani-Hamed, A.G. Cohen, E. Katz and A.E. Nelson, The littlest Higgs, JHEP 07 (2002) 034 [hep-ph/0206021] [SPIRES].

[2] M. Schmaltz and D. Tucker-Smith, Little Higgs Review, Ann. Rev. Nucl. Part. Sci. 55 (2005) 229 [hep-ph/0502182] [SPIRES]. 
[3] M. Perelstein, Little Higgs models and their phenomenology, Prog. Part. Nucl. Phys. 58 (2007) 247 [hep-ph/0512128] [SPIRES].

[4] H. Georgi, The Higgs as a pseudo-Goldstone boson, Comptes Rendus Physique 8 (2007) 1029 [SPIRES].

[5] D.B. Kaplan and H. Georgi, $\mathrm{SU}(2) \times \mathrm{U}(1)$ Breaking by Vacuum Misalignment, Phys. Lett. B 136 (1984) 183 [SPIRES].

[6] D.B. Kaplan, H. Georgi and S. Dimopoulos, Composite Higgs Scalars, Phys. Lett. B 136 (1984) 187 [SPIRES].

[7] T. Han, H.E. Logan, B. McElrath and L.-T. Wang, Phenomenology of the little Higgs model, Phys. Rev. D 67 (2003) 095004 [hep-ph/0301040] [SPIRES].

[8] C. Csáki, J. Hubisz, G.D. Kribs, P. Meade and J. Terning, Big corrections from a little Higgs, Phys. Rev. D 67 (2003) 115002 [hep-ph/0211124] [SPIRES].

[9] J.L. Hewett, F.J. Petriello and T.G. Rizzo, Constraining the littlest Higgs. ((U)), JHEP 10 (2003) 062 [hep-ph/0211218] [SPIRES].

[10] C. Csáki, J. Hubisz, G.D. Kribs, P. Meade and J. Terning, Variations of little Higgs models and their electroweak constraints, Phys. Rev. D 68 (2003) 035009 [hep-ph/0303236] [SPIRES].

[11] R. Barbieri, A. Pomarol, R. Rattazzi and A. Strumia, Electroweak symmetry breaking after LEP1 and LEP2, Nucl. Phys. B 703 (2004) 127 [hep-ph/0405040] [SPIRES].

[12] S. Chang and J.G. Wacker, Little Higgs and custodial SU(2), Phys. Rev. D 69 (2004) 035002 [hep-ph/0303001] [SPIRES].

[13] S. Chang, A 'littlest Higgs' model with custodial SU(2) symmetry, JHEP 12 (2003) 057 [hep-ph/0306034] [SPIRES].

[14] H.-C. Cheng and I. Low, TeV symmetry and the little hierarchy problem, JHEP 09 (2003) 051 [hep-ph/0308199] [SPIRES].

[15] H.-C. Cheng and I. Low, Little hierarchy, little Higgses and a little symmetry, JHEP 08 (2004) 061 [hep-ph/0405243] [SPIRES].

[16] H.-C. Cheng, I. Low and L.-T. Wang, Top partners in little Higgs theories with T-parity, Phys. Rev. D 74 (2006) 055001 [hep-ph/0510225] [SPIRES].

[17] B. Grinstein, R. Kelley and P. Uttayarat, Hidden fine tuning in the quark sector of little Higgs models, JHEP 09 (2009) 040 [arXiv:0904.1622] [SPIRES].

[18] J. Preskill, Subgroup alignment in hypercolor theories, Nucl. Phys. B 177 (1981) 21 [SPIRES].

[19] M.E. Peskin, The Alignment of the Vacuum in Theories of Technicolor, Nucl. Phys. B 175 (1980) 197 [SPIRES].

[20] M. Perelstein, M.E. Peskin and A. Pierce, Top quarks and electroweak symmetry breaking in little Higgs models, Phys. Rev. D 69 (2004) 075002 [hep-ph/0310039] [SPIRES].

[21] B. Grinstein and M. Trott, Top quark induced vacuum misalignment in little Higgs models, JHEP 11 (2008) 064 [arXiv:0808.2814] [SPIRES].

[22] H. Georgi, Weak Interactions And Modern Particle Theory, Benjamin/cummings, Menlo Park, U.S.A. (1984) [SPIRES]. 
[23] T.P. Cheng, E. Eichten and L.-F. Li, Higgs Phenomena in Asymptotically Free Gauge Theories, Phys. Rev. D 9 (1974) 2259 [SPIRES].

[24] M.E. Machacek and M.T. Vaughn, Two Loop Renormalization Group Equations in a General Quantum Field Theory. 1. Wave Function Renormalization, Nucl. Phys. B 222 (1983) 83 [SPIRES].

[25] J. Gasser and H. Leutwyler, Chiral Perturbation Theory to One Loop, Ann. Phys. 158 (1984) 142 [SPIRES].

[26] J. Gasser and H. Leutwyler, Chiral Perturbation Theory: Expansions in the Mass of the Strange Quark, Nucl. Phys. B 250 (1985) 465 [SPIRES].

[27] J.R. Espinosa and J.M. No, Scalar Loops in Little Higgs Models, JHEP 01 (2007) 006 [hep-ph/0610255] [SPIRES]. 